\title{
Arginine enzymatic deprivation and diet restriction for cancer treatment
}

\author{
Wissam Zam* \\ Al-Andalus University for Medical Sciences, Faculty of Pharmacy, Analytical and Food Chemistry, Tartous, \\ Syrian Arab Republic
}

\begin{abstract}
Recent findings in amino acid metabolism and the differences between normal, healthy cells and neoplastic cells have revealed that targeting single amino acid metabolic enzymes in cancer therapy is a promising strategy for the development of novel therapeutic agents. Arginine is derived from dietary protein intake, body protein breakdown, or endogenous de novo arginine production and several studies have revealed disturbances in its synthesis and metabolism which could enhance or inhibit tumor cell growth. Consequently, there has been an increased interest in the arginine-depleting enzymes and dietary deprivation of arginine and its precursors as a potential antineoplastic therapy. This review outlines the most recent advances in targeting arginine metabolic pathways in cancer therapy and the different chemo- and radio-therapeutic approaches to be co-applied.
\end{abstract}

Key words: Arginine-depleting enzyme/antineoplastic therapy. Dietary deprivation.

\section{INTRODUCTION}

Certain cancers may be auxotrophic for a particular amino acid, and amino acid deprivation is one method to treat these tumors. The strategy of enzymatic degradation of amino acids to deprive malignant cells of important nutrients is an established component of induction therapy of several tumor cells.

The amino acid arginine has considerable nutritional and physiological significance as it is recognized as an important precursor for the synthesis of proteins, urea, and creatine as well as for the synthesis of signaling molecules such as glutamate, nitric oxide, and agmatine (Wu, Morris, 1998). Although arginine is a dispensable (nonessential) amino acid for healthy humans, it is conditionally essential under certain physiological conditions or disease state (Roos, Loos, 1973; Rose, Haines, Warner, 1954; Barbul, 1986).

For years, depletion of arginine has been shown to be an effective and promising anti-cancer treatment in vitro and in vivo (Bach, Swaine, 1965; Wheatley, 2005). By culturing cells in the media depleted of arginine, a

\footnotetext{
*Correspondence: W. Zam. Al-Andalus University for Medical Sciences, Faculty of Pharmacy, Analytical and Food Chemistry, Tartous, Syrian Arab Republic. Tel: +932933724703. E-mail: w.zam@au.edu.sy
}

variety of human cancer cells have been found to be auxotrophic for arginine, depletion of which results in cell death (Tytell, Neuman, 1960; Kraemer, 1964; Dillon et al., 2004). Arginine can be degraded by three enzymes: arginase, arginine decarboxylase and arginine deiminase (ADI). Both arginine decarboxylase and ADI are not expressed in mammalian cells (Morris, 2007; Miyazaki et al., 1990).

Arginine withdrawal leads to increased protein turnover-via reduced synthesis and increased breakdown [suppression of mammalian target of rapamycin (mTOR) and proteosomal degradation, respectively]-and triggers caspase-dependent and caspase-independent apoptotic cell death in a cell type-dependent manner (Kim et al., 2009).

This review focus on the recent development of targeted therapy of a subset of human malignancies with altered arginine metabolism by enzymatic degradation or diet restriction.

\section{Enzymatic degradation of arginine}

\section{Arginine deiminase (ADI)}

Arginine deiminase (ADI) is a microbial enzyme from mycoplasma, it has high affinity to arginine and catalyzes arginine to citrulline and ammonia. Citrulline can be recycled back to arginine in normal cells which 
express argininosuccinate synthetase (ASS), whereas ASS(-) tumor cells cannot. Loss of ASS expression has been most widely associated with melanoma and hepatocellular carcinoma, although it has now been identified in other tumor types including pancreatic cancer, leukemia, prostate cancer and renal cell carcinoma (Gong et al., 2000; Ensor et al., 2002; Yoon et al., 2007; Bowles et al., 2008; Kim et al., 2009).

As the bacterial enzyme is highly immunogenic in humans, therapeutic preparations of ADI have been conjugated with polyethylene glycol (20000 Da; ADIPEG20) that serves to reduce the immunogenicity of the enzyme while greatly improving its pharmacokinetic halflife in serum (Feun, Savaraj, 2006; Feun et al., 2008; Ni, Schwaneberg, Sun, 2008).

Clinical trials of ADI-PEG20 have been followed in both hepatocellular carcinoma and melanoma. Pharmacokinetic and pharmacodynamic results from these studies demonstrated that a dose of $160 \mathrm{IU} \mathrm{m} 2$ ADI-PEG20 was sufficient to reduce plasma arginine levels from $\sim 130 \mu \mathrm{m}$ to below the level of detection $(<2 \mu \mathrm{m})$ for at least 7 days (Ascierto et al., 2005; Delman et al., 2005; Izzo et al., 2004). However, arginine deprivation can also induce ASS expression in certain melanoma cell lines which can lead to in vitro drug resistance (Feun et al., 2008).

Acute myeloid leukemia (AML) cells from most patients with AML are deficient in ASS1. ADI-PEG 20 alone induced responses in 19 of 38 AMLs in vitro and 3 of 6 AMLs in vivo, leading to caspase activation in sensitive AMLs. ADI-PEG 20-resistant AMLs showed higher relative expression of ASS1 than sensitive AMLs (Miraki-Moud et al., 2015). Results also showed that the other enzyme in the arginine synthesis pathway, argininosuccinate lyase (ASL), was also more highly expressed in ADI-PEG 20-resistant AMLs (Miraki-Moud et al., 2015).

Patients with small cell lung cancer (SCLCs) often demonstrate a robust initial response to chemotherapy; relapse rates remain high, highlighting the need for the development of novel therapeutic options in this disease. A recent study showed that a large proportion of SCLCs lack the expression of ASS, where $\sim 50 \%$ of human tumors examined were found to lack expression of the enzyme (Jungbluth et al., 2010). In vitro studies using both adherent and non-adherent ASS-deficient SCLC cell lines demonstrated that ADI-PEG20 caused dose-dependent anti-proliferative efficacy by inducing autophagy, followed by caspase-independent cell death (Kelly et al., 2012). Additionally, prolonged treatment with ADI-PEG20 may lead to the induction of ASS expression and the activation of other cellular pathways associated with resistance to apoptosis, possibly limiting the overall treatment window for ADI (Kim et al., 2009; Tsai et al., 2009). SCLCs xenografts treated continuously with 5 IU ADI-PEG20 study demonstrated a sustained response to ADI-PEG20 for the 90-day period of the study, suggesting that at least at this dose, profound resistance to the anti-proliferative effect of ADI-PEG20 had not been induced (Kelly et al., 2012).

ASS1 negativity was detected in $40 \%$ of bladder cancers as indicated by Allen et al. (2014) ADI-PEG20 was synthetically lethal in ASS1-methylated bladder cells and its exposure was associated with a marked reduction in intracellular levels of thymidine, due to suppression of both uptake and de novo synthesis. Notably, inhibition of de novo synthesis was associated with potentiation of ADI-PEG20 activity by the antifolate drug pemetrexed (Allen et al., 2014).

Renal cell carcinoma (RCC) cells treated with ADI showed a low expression level of ASS and remarkable growth retardation in a dose dependent manner. Histological examination of the tumors revealed that tumor angiogenesis and vascular endothelial growth factor (VEGF) expression were significantly diminished by ADI administration (Yoon et al., 2007).

Bowles et al. (2008) evaluated the level of ASS expression in 47 human pancreatic adenocarcinoma specimens and 20 normal pancreas biopsies. ASS expression was not detected in 41 of the 47 (87\%) pancreatic adenocarcinoma specimens and was observed to varying degrees in the non-neoplastic pancreatic ductal cells (Bowles et al., 2008). ASS mRNA expression was observed at varying levels with L3.3 cell lines having the highest level of expression while MIA-PaCa-2 and PANC1 having very low levels. All cell lines were subjected to increasing doses of PEG-ADI. Cell growth was inhibited in MIA-PaCa- 2 cells, with an IC50 of $0.3 \mu \mathrm{g} / \mathrm{mL}$ while L3.3 cells were unaffected by a broad dose-range of PEGADI. The cytotoxic response of PANC-1 to PEG-ADI was very similar to that of MIA-PaCa-2, with the same IC50. The growth kinetics of the 3 pancreatic cancer cell lines was then examined after ADI treatment $(0.3 \mu \mathrm{g} / \mathrm{mL})$ (Bowles et al., 2008).

It was also observed that the development of chemoresistance to platinum compounds in ovarian carcinomas leads to collateral appearance of arginine auxotrophy due to the downregulation of ASS (Nicholson et al., 2009), adding these tumors to the list of potential targets of arginine deprivation-based enzymotherapy. Arginine deprivation in fact depressed the activation of the signaling kinases 4E-BP1 and p70-S6k that govern the protein synthesis pathway. These kinases are downstream 
of mTOR (Hara et al., 1998), which also negatively controls autophagy. Shuvayeva et al. (2014) showed that combinational treatment based on arginine deprivation and an autophagy inhibitor (e.g., chloroquine, a known nontoxic antimalarial drug) can potentially be applied as a second line treatment for a subset of ovarian carcinomas deficient in ASS (Shuvayeva et al., 2014).

Results indicated that ADI-PEG20 can effectively induce cell death in prostate cancer cells with low (PC3 cells) or absent (CWR22Rv1 cells) ASS expression. The effect of ADI-PEG20 in CWR22Rv1 mouse xenografts reveals reduced tumor activity and reduced tumor growth as a monotherapy and in combination with chemotherapeutic agents such as docetaxel or autophagy inhibitors such as chloroquine. In contrast, $\mathrm{LNCaP}$ cells highly express ASS and are therefore resistant to both ADI-PEG20 and autophagic inhibition (Kim et al., 2009).

\section{Recombinant human arginase (rhArg)}

Recombinant human arginase (rhArg) has been developed for arginine deprivation therapy in cancer, and is currently under clinical investigation. During pre-clinical evaluation, rhArg has exhibited significant anti-proliferative activity in cancer cells deficient in the expression of ornithine carbamoyl transferase (OCT) which metabolizes ornithine and carbamoly phosphate into citrulline (Hsueh et al., 2012). OCT is highly expressed in liver and small intestine, and catabolizes the conversion of ornithine to citrulline (Raijman, 1974). However, OCT expression in cancer and other normal tissues is mostly down-regulated due to epigenetic changes such as DNA hypermethylation (Delers et al., 1984).

Recombinant human arginase (rhArg) demonstrated significant cytotoxicity in hepatocellular carcinoma and melanoma, in vitro and in vivo (Cheng et al., 2007; Lam et al., 2009; Lam et al., 2010). In contrast to ADI-PEG20, the sensitivity to rhArg in hepatocellular carcinoma and melanoma is independent of ASS expression.

Quantitative real-time PCR showed minimal to absent gene expression of OCT, but ample expression of ASS expression in 3 cell lines of in human prostate cancer cells: LNCaP (androgen-dependent), PC-3 and DU-145 (both androgen-independent). Cell viability assay after 72-h exposure of rhArg showed all 3 lines had half maximal inhibitory concentration less than or equal to 0.02 $\mathrm{U} / \mathrm{ml}$. Addition of ornithine to cell culture media failed to rescue these cells from rhArg-mediated cytotoxicity. Decreased phosphorylation of 4E-BP1, a downstream effector of mammalian target of rapamycin (mTOR), was noted in DU-145 and PC-3 after exposure to rhArg.
Moreover, there was no significant apoptosis induction after arginine deprivation by rhArg in all 3 prostate cancer cell lines. In conclusion, rhArg demonstrates a promising novel agent for prostate cancer treatment (Cheng et al., 2007).

To overcome the short circulatory half-life of arginase, $\operatorname{rhArg}(\mathrm{Mn})$-PEG5000 was developed and investigated with in vivo and in vitro studies in $\mathrm{HCC}$ (Lam et al., 2010). RhArg(Mn)-PEG5000 was effective in inhibiting growth of HCC cell lines as well as melanoma cell lines, with IC50 ranging from 0.1-2 IU/ $\mathrm{ml}$. OTC-negative cancer cell lines were more sensitive to $\operatorname{rh} \operatorname{Arg}(\mathrm{Mn})-\mathrm{PEG} 5000$, while OTC-transfected cell lines were resistant. Since some OTC-negative cell lines that were sensitive to $\operatorname{rhArg}(\mathrm{Mn})$-PEG5000 had ASS expression, this arginase may be an alternative for the cancer patient with an ASS-expressing tumor (Yoon et al., 2013).

Effectiveness of rhArg was ameliorated by replacing the two $\mathrm{Mn}^{2+}$ ions normally present in human arginase I with $\mathrm{Co}^{2+}$ resulted in a significantly lowered $K M$ value without a concomitant reduction in $k$ cat which increased the catalytic efficiency. In addition, the $\mathrm{pH}$ dependence of the reaction was shifted from a pKa of 8.5 to a pKa of 7.5 close to the $\mathrm{pH}$ of human plasma. Just as important for improved efficacy, $\mathrm{Co}^{2+}$ substitution leads to significantly increased serum stability of the enzyme (Stone et al., 2010). In vitro cytotoxicity experiments showed that the $\mathrm{Co}^{2+}$ substituted rhArg displays an approximately 12-15-fold lower IC50 value for the killing of human hepatocellular carcinoma Hep3b and melanoma A375 cell lines (Stone et al., 2010).

It was also showed that weekly treatment of $8 \mathrm{mg} /$ kg Co-hArgI-PEG effectively controlled Panc-1 (PC) tumor xenografts by inducing autophagy and apoptosis in vitro with significant increased expression of activated caspase-3 (Glazer et al., 2011).

\section{Arginine decarboxylase}

Arginine decarboxylase (ADC) catalyzes the decarboxylation of arginine to agmatine and carbon dioxide (Leung, Wei, 2012). So, some researchers hypothesize that by applying ADC to the tumor cells, the arginine can be depleted and hence the growth of tumor cells will be inhibited. Leung and Wei treated colorectal cancer cell lines (HCT116 and LoVo) with recombinant ADC (rADC) for 72 hours. The anti-proliferation effect of rADC was determined by MTT assay and the effect of rADC on cell cycle re-distribution and apoptosis was analyzed by flow cytometry. Results indicated that rADC 
exhibited an in vitro anti-proliferation effect on both colorectal cell lines tested. Upon treatment with rADC, tumor cells were found to arrest at S-phase of the cell cycle. rADC also had a potent apoptosis-inducing effect on both cell lines. Further studies showed that rADC-induced apoptosis was caspase- 3 dependent and might follow the mitochondrial apoptotic pathway (Leung, Wei, 2012).

Philip, Campbell and Wheatley (2003) tested the use of arginine decarboxylase from $E$. coli in human diploid fibroblasts, human uterus HeLa and murine leukemia L1210 cells. The response of HeLa to arginine decarboxylase was very similar to that seen in fibroblast cultures, with good inhibition seen at 1 unit $\mathrm{mL}^{-1}$, but more immediate and obvious cell death occurred within 4 days with 5 units $\mathrm{mL}^{-1}$. Recovery with citrulline was effective, whereas adding arginine to cultures treated with enzymes failed to rescue growth because, unlike citrulline, it was quickly degraded (Philip, Campbell, Wheatley, 2003). L1210 cell line has a considerably higher nutrient demand than HeLa cells, and a far greater one than fibroblasts. The dose-response curve for arginine decarboxylase shows its considerable efficacy against L1210 cells after 3 days treatment. Most cells were irrecoverable after 4 days of arginine free medium treatment. A few cells persisted, as shown by microscopic examination, these being predominantly large multinucleated cells, as seen with HeLa cell cultures. Citrulline rescued the cells under arginine decarboxylase treatment, but arginine could not (Philip, Campbell, Wheatley, 2003).

While in some respects arginine decarboxylase could be an attractive enzyme to use, some problems challenged this use. The enzyme produces agmatine which had toxic effects when it exceeded millimolar levels in the absence of arginine, and therefore some doubt remains as to whether a build-up under such conditions is accompanied by toxic sequelae in the body. However, agmatine is a small molecule that might be quickly cleared but it is also known to inhibit nitric oxide synthase and lead to loss of vascular tone and signaling in many other vital functions (Galea et al., 1996). However, new evidence strongly suggests that a special arginine pool may be generated, which is closely linked or channeled with nitric oxide synthase in appropriate cells (Flam et al., 2001).

Furthermore, this enzyme requires pyridoxal-5phosphate as a cofactor, but in culture researchers have found good activity in media where no additional cofactor was supplied (Galea et al., 1996).

In general, recombinant human arginine decarboxylase produced by E. coli is considerably more active than enzymes prepared from natural straightforward enzyme extraction from other sources, both in vivo and in vitro. However, pegylated arginine decarboxylase loses almost all its activity, probably because it has far fewer lysine residues than arginase. While this will diminish its potential use in vivo, this enzyme still requires to be more fully evaluated (Philip, Campbell, Wheatley, 2003).

\section{Difluoromethylornithine}

Polyamines are organic compounds found in every living cell. They are synthesized endogenously from the arginine-derived product ornithine which is converted by ODC to the diamine putrescine, which is sequentially converted into the polyamines spermidine and spermine. Polyamines regulate oncogene expression and function through transcriptional and posttranscriptional processes which implicate a specific link between polyamines and cancer (Bachrach, Wang, Tabib, 2001).

Dietary arginine enhances the risk of APCdependent colon carcinogenesis in mouse models by a mechanism involving polyamines synthesis. The primary consequence of dietary arginine is to increase the adenoma grade in these mice. DFMO (difluoromethylornithine), an irreversible inhibitor of ornithine decarboxylase, inhibits dietary arginine-induced colon carcinogenesis in $\mathrm{C} 57 \mathrm{BL} / 6 \mathrm{~J}-A p c M i n / \mathrm{J}$ mice by suppressing the arginineinduced increase in adenoma grade (Gerner, 2007; Yerushalmi et al., 2006).

DFMO has been tested in clinical trials for children with neuroblastoma which is the most common solid malignancy in children and has generally a poor prognosis. Current trial designs include testing lower dose DFMO alone $(2,000 \mathrm{mg} / \mathrm{m} 2 /$ day $)$ starting at the completion of standard therapy, or higher doses combined with chemotherapy (up to $9,000 \mathrm{mg} / \mathrm{m} 2 /$ day) for patients with relapsed disease that has progressed (Bassiri et al., 2015). The findings revealed minimal side effects in patients as well three patients with long-term survival. Sholler and the Neuroblastoma and Medulloblastoma Translational Research Consortium are now testing DFMO in a Phase II clinical trial to prevent neuroblastoma relapse (Sholler et al., 2015).

\section{Attenuation of Argininosuccinate Lyase}

The conversion from citrulline to arginine involves two enzymes, argininosuccinate synthetase (ASS) and argininosuccinate lyase (ASL). ASS seems to be the rate-limiting step in the production of arginine (Husson et al., 2003), but citrulline availability controls the endogenous levels of arginine and NO throughout the body (Delage et al., 2010). ASL is transcriptionally induced 
by endoplasmic reticulum stress and is overexpressed in some human liver tumors. Downregulation of ASL by short hairpin RNA (shRNA) in three liver cancer cell lines, ML-1, HuH-7, and HepG2, decreased colony formation in vitro and tumor growth in vivo. The inhibition was mainly mediated by a decrease of cyclin A2 and NO without altering significantly the total cellular arginine level (Huang et al., 2013).

While exploring the same effect in breast cancer tissues, researchers found that ASL was induced by endoplasmic reticulum stress and was significantly upregulated in breast cancer tissues compared to that in the corresponding normal tissues. Downregulation of ASL inhibited the growth of breast cancer in vitro and in vivo by reducing both cyclin $\mathrm{A} 2$ and $\mathrm{NO}$, and delaying $\mathrm{G} 2 / \mathrm{M}$ transition (Huang et al., 2015).

\section{Arginine and protein modified diet}

Castillo et al. (1994) have studied the changes in whole body arginine metabolism over a 6-day argininefree diet (Castillo et al., 1994) and extended the period of dietary restriction to 4 weeks, including limiting the intakes of the major, immediate dietary precursors of arginine (glutamate, proline, and aspartate) (Yu et al., 2001). In a response to this restriction arginine homeostasis is maintained. Results indicate a reduced rate of arginine oxidation with maintenance of endogenous arginine synthesis, which occurs at a rate of about onethird of the dietary intake level (Tharakan et al., 2008). However, the present diet contained alanine and serine that presumably serve as adequate sources of $\alpha$-amino N. It is to be considered that these results can be applied only to healthy subjects and with nutritional balance, whereas a source of dietary arginine is required in severely stressed patients and so in this case arginine is a conditionally indispensable amino acid ( $\mathrm{Yu}$ et al., 2001). Female mice with skin carcinogenesis were placed on a special synthetic amino acid diet deficient in arginine. This diet exhibited a $40-50 \%$ reduction in tumor multiplicity and had a significantly lower tumor incidence throughout a 19-week promotion period. The addition of ornithine completely reversed the reduction in the rate and extent of tumorigenesis in the arginine-free animals (Gonzalez, Byus, 1991).

Dietary restricted protein implies a reduction in arginine intake. Fontana et al. (2013) examined the effects of isocaloric modifications in dietary protein quantity or quality on tumor growth in the human xenograft LuCaP23.1, LuCaP35V prostate cancer models and WHIM16 breast cancer models. Results showed a 70\% inhibition of tumor growth in the castrate-resistant LuCaP23.1 prostate cancer model and a 56\% inhibition in the WHIM16 breast cancer model fed with a $7 \%$ protein diet when compared to an isocaloric $21 \%$ protein diet. This inhibitory effect was associated with a reduction in serum PSA and IGF-1 levels, and a down-regulation of intra-tumor mTOR activity in the LuCaP23.1 and LuCaP35V models. Protein restriction was also associated with modulation of specific histone markers suggesting epigenetic modulation (Fontana et al., 2013).

\section{Combination therapies}

Although metabolic enzymotherapy based on arginine deprivation is considered as nontoxic and selective, it is not free of certain limitations. One such limitation arises from the upregulation of ASS expression in many tumors in response to arginine starvation, leading to the appearance of the ASS-positive tumor relapse insensitive to the therapy (Vynnytska-Myronovska et al., 2012). Also, results showed that tumor cells become profoundly more resistant to arginine withdrawal in in vitro 3D spheroid models relative to respective monolayer cultures (Vynnytska-Myronovska et al., 2013). This phenomenon is consistent with the results of animal studies and ongoing clinical trials which showed that arginine deprivation is effective in inhibiting tumor growth but not in inducing tumor regression. The latter observation stimulates further search for more efficient rational combinational therapeutic approaches based on arginine deprivation.

Malignant pleural mesothelioma (MPM) cells do not express argininosuccinate synthetase (ASS), and thus are vulnerable to ADI-PEG20. Concomitant treatment using $50 \mathrm{ng} / \mathrm{mL}$ of ADI-PEG20 and $10 \mathrm{ng} / \mathrm{mL}$ of TNF-related apoptosis-inducing ligand (TRAIL) for $24 \mathrm{~h}$ resulted in profound cell death with $67 \%$ of cells positive for caspase- 3 activity, while ADI-PEG20 alone or TRAIL alone resulted in only $10-15 \%$ cell death. This positive amplification loop is mediated through the cleavage of proapototic protein "BID" (Wangpaichitr et al., 2014). Savaraj et al. (2010) have found that combination of ADI-PEG20 (0.05 $\mu \mathrm{g} /$ $\mathrm{mL})$ with cisplatin $(0.1 \mu \mathrm{g} / \mathrm{mL})$ can increase apoptosis in melanoma cell lines which may be partly due to increase in DNA damage (Savaraj et al., 2010). Miraki-Moud et al. (2015) showed that the combination of ADI-PEG 20 and cytarabine chemotherapy was more effective than either treatment alone resulting in responses in all AMLs tested in vivo regardless of ASS1 expression (Miraki-Moud et al., 2015). Interestingly, the addition of $5 \mathrm{uM}$ MEK inhibitor (U0126) to $0.06 \mathrm{ug} / \mathrm{mL}$ of ADI-PEG20 increased both 
growth inhibitory effect and apoptosis in the treatment of a melanoma cell line, Mel1220. Growth inhibitory effect in this combination was $80 \%$, while it was only $15 \%$ with ADI-PEG20 alone and 25\% with U0126 alone. The same combination produced $26 \%$ of cells which are positive for caspase activity and 30\% cell death (Savaraj et al., 2010). Phase I study examined the toxicity and tolerability, of weekly intramuscular injection ranging from 4.5-36 $\mathrm{mg} / \mathrm{m}^{2}$ of ADI-PEG 20 in combination with $75 \mathrm{mg} / \mathrm{m}^{2}$ of docetaxel every three weeks in patients with advanced solid malignancies. ADI-PEG 20 demonstrated reasonable toxicity in combination with docetaxel. Arginine was variably suppressed with more than half patients achieving at least a $50 \%$ reduction in baseline values. In 14 patients with evaluable disease, four partial responses (including two patients with PSA response) were documented and seven patients had stable disease (Tomlinson et al., 2015).

Results have shown that the use of rhArg treatment resulted in the appearance of autophagosomes and upregulation of microtubule-associated protein light chain $3 \mathrm{II}$, indicating that rhArg induced autophagy in lymphoma cells. So, blocking autophagy using pharmacological inhibitors such as 3-methyladenine and chloroquine enhanced the cell killing effect of rhArg (Zeng et al., 2013).

A new combinatorial metabolic-chemo-radiotreatment regime was proposed by Vynnytska-Myronovska based on arginine starvation, low doses of arginine natural analog canavanine and irradiation. Combination treatment was remarkably efficient. In particular, pretreatment of cancer cells with the arginine-degrading enzyme arginase combined with or without low concentration of canavanine substantially enhanced cell radioresponse reflected by a loss in spheroid regrowth probability and $\operatorname{SCD}(50)$ values reduced by a factor of 1.5-3 (Vynnytska-Myronovska et al., 2012). Gong et al. demonstrated that arginine deiminase suppresses the growth of unfavorable experimental neuroblastomas and that this effect is potentiated by irradiation. This combination leads to a reduction in the absolute number of tumor vessels and of perfused tumor vessels without increasing tumor hypoxia (Gong et al., 2003). Researchers have evaluated ASS-1 protein pattern and the impact of treating 3-D cultures of head and neck squamous cell carcinoma (HNSCC) with irradiation under arginine-deficient conditions by rh-arginase. Results showed that all HNSCC cell lines express ASS-1 protein but show different intrinsic levels. Independent of that, arginine deprivation leads to an acute cell growth arrest in all 2-D and 3-D cultures and only reduced regrowth capacity upon completion of the diet in 2-D cultures. Similarly, no volume growth during starvation was observed for the two 3-D culture models studied. Arginine withdrawal resulted in a strong radiosensitization in SAS spheroids (Kuo, Savaraj, Feun, 2010).

\section{CONCLUSION}

Arginine can be catabolized by three enzymes: arginase, arginine decarboxylase and arginine deiminase. So the use of these enzymes is a promising procedure for arginine enzymatic deprivation as a targeted approach to the treatment of certain tumors. Pegylated forms of arginine deiminase is used in tumors deficient in ASS, while rhArg has exhibited significant anti-proliferative activity in cancer cells deficient in the expression of OCT which is mostly down-regulated in cancer tissues due to epigenetic changes such as DNA hypermethylation. Effectiveness of rhArg was ameliorated by replacing the two $\mathrm{Mn}^{2+}$ ions normally present in human arginase I with $\mathrm{Co}^{2+}$. The anti-proliferation effect of rADC was determined in human diploid fibroblasts, human uterus HeLa, colorectal and murine leukemia L1210 cells but this enzyme still requires to be more fully evaluated.

DFMO (difluoromethylornithine), an irreversible inhibitor of ornithine decarboxylase, inhibits polyamines synthesis and shows efficacy in colon carcinogenesis and neuroblastoma. Furthermore, downregulation of ASL inhibited the growth of breast cancer and liver cancer cell lines in vitro and in vivo.

The long-term use of ADI-PEG20 treatment induces ASS expression in certain melanoma cell lines which can lead to in vitro drug resistance. This form of resistance may be overcome by using chemo co-applied agents such as cytarabine and cisplatin or by the use of rhArg as an alternative for the cancer patient with an ASS-expressing tumor. Autophagy is the most important side effect diminishing the efficacy rhArg which is counteracted by the use of pharmacological inhibitors such as 3-methyladenine and chloroquine. Many combinatorial metabolic-radio-treatment regimens were proposed and results showed that arginine withdrawal resulted in a strong radiosensitization.

Arginine metabolism differs greatly between healthy subjects and stressed patients. It is to be considered that in a response to a dietary restriction of arginine, homeostasis is maintained in healthy subjects whereas a source of dietary arginine is required in severely stressed patients and so in this case arginine is a conditionally indispensable amino acid. So, dietary restricted protein implies a reduction in arginine intake which can be a future prospective method used in combination with arginine enzymatic degradation for the reduction in the rate and extent of tumorigenesis. 


\section{REFERENCES}

Allen MD, Luong P, Hudson C, Leyton J, Delage B, Ghazaly E, et al. Prognostic and therapeutic impact of argininosuccinate synthetase 1 control in bladder cancer as monitored longitudinally by PET imaging. Cancer Res. 2014;74(3):896-907.

Ascierto PA, Scala S, Castello G, Daponte A, Simeone E, Ottaiano A, et al. Pegylated arginine deiminase treatment of patients with metastatic melanoma: results from phase I and II studies. J Clin Oncol. 2005;23(30):7660-7668.

Bach SJ, Swaine D. The effect of arginase on the retardation of tumour growth. Br J Cancer. 1965;19(2):379-386.

Bachrach U, Wang YC, Tabib A. Polyamines: new cues in cellular signal transduction. News Physiol Sci. 2001;16:106109.

Barbul A. Arginine: biochemistry, physiology, and therapeutic implications. JPEN J Parenter Enteral Nutr. 1986;10(2):227-238.

Bassiri H, Benavides A, Haber M, Gilmour SK, Norris MD, Hogarty MD. Translational development of difluoromethylornithine (DFMO) for the treatment of neuroblastoma. Transl Pediatr. 2015;4(3):226-238.

Bowles TL, Kim R, Galante J, Parsons CM, Virudachalam $\mathrm{S}$, Kung HJ, et al. Pancreatic cancer cell lines deficient in argininosuccinate synthetase are sensitive to arginine deprivation by arginine deiminase. Int J Cancer. 2008;123(8):1950-1955.

Castillo L, Ajami A, Branch S, Chapman TE, Yu YM, Burke $\mathrm{JF}$, et al. Plasma arginine kinetics in adult man: response to an arginine-free diet. Metabolism. 1994;43(1):114-120.

Cheng PN, Lam TL, Lam WM, Tsui SM, Cheng AW, Lo WH, et al. Pegylated recombinant human arginase (rhArgpeg $5,000 \mathrm{mw}$ ) inhibits the in vitro and in vivo proliferation of human hepatocellular carcinoma through arginine depletion. Cancer Res. 2007;67(1):309-317.

Delage B, Fennell DA, Nicholson L, McNeish I, Lemoine NR, Crook T. Arginine deprivation and argininosuccinate synthetase expression in the treatment of cancer. Int J Cancer. 2010;126(12):2762-2772.

Delers A, Szpirer J, Szpirer C, Saggioro D. Spontaneous and 5 -azacytidine-induced reexpression of ornithine carbamoyl transferase in hepatoma cells. Mol Cell Biol. 1984;4(4):809-812.
Delman KA, Brown TD, Thomas M, Ensor CM, Holtsberg FW, Bomalaski JS, et al. Phase I/II trial of pegylated arginine deiminase (ADI-PEG20) in unresectable hepatocellular carcinoma. J Clin Oncol (Meeting Abstracts). 2005;23(16):4139413.

Dillon BJ, Prieto VG, Curley SA, Ensor CM, Holtsberg FW, Bomalaski JS, et al. Incidence and distribution of argininosuccinate synthetase deficiency in human cancers: a method for identifying cancers sensitive to arginine deprivation. Cancer. 2004;100(4):826-833.

Ensor CM, Holtsberg FW, Bomalaski JS, Clark MA. Pegylated arginine deiminase (ADI-SS PEG20,000 mw) inhibits human melanomas and hepatocellular carcinomas in vitro and in vivo. Cancer Res. 2002;62(19):5443-5450.

Feun L, Savaraj N. Pegylated arginine deiminase: a novel anticancer enzyme agent. Expert Opin Investig Drugs. 2006;15(7):815-822.

Feun L, You M, Wu CJ, Kuo MT, Wangpaichitr M, Spector S, Savaraj N. Arginine deprivation as a targeted therapy for cancer. Curr Pharm Des. 2008;14(11):1049-1057.

Flam BR, Hartmann PJ, Harrell-Booth M, Solomonson LP, Eichler DC. Caveolar localization of arginine regeneration enzymes, argininosuccinate synthase, and lyase, with endothelial nitric oxide synthase. Nitric Oxide. 2001;5(2):187-197.

Fontana L, Adelaiye RM, Rastelli AL, Miles KM, Ciamporcero E, Longo VD, et al. Dietary protein restriction inhibits tumor growth in human xenograft models of prostate and breast cancer. Oncotarget. 2013;4(12):2451-2461.

Galea E, Regunathan S, Eliopoulos V, Feinstein DL, Reis DJ. Inhibition of mammalian nitric oxide synthases by agmatine, an endogenous polyamine formed by decarboxylation of arginine. Biochem J. 1996;316(Pt.1):247-249.

Gerner EW. Impact of dietary amino acids and polyamines on intestinal carcinogenesis and chemoprevention in mouse models. Biochem Soc Trans. 2007;35(2):322-325.

Glazer ES, Stone EM, Zhu C, Massey KL, Hamir AN, Curley SA. Bioengineered Human Arginase I with Enhanced Activity and Stability Controls Hepatocellular and Pancreatic Carcinoma Xenografts. Translational Oncology. 2011;4(3):138-146. 
Gong H, Pöttgen C, Stüben G, Havers W, Stuschke M, Schweigerer L. Arginine deiminase and other antiangiogenic agents inhibit unfavorable neuroblastoma growth: Potentiation by irradiation. Int J Cancer. 2003;106(5):723-728.

Gong H, Zolzer F, von Recklinghausen G, Havers W, Schweigerer L. Arginine deiminase inhibits proliferation of human leukemia cells more potently than asparaginase by inducing cell cycle arrest and apoptosis. Leukemia. 2000;14(5):826-829.

Gonzalez GG, Byus CV. Effect of dietary arginine restriction upon ornithine and polyamine metabolism during twostage epidermal carcinogenesis in the mouse. Cancer Res. 1991;51(11):2932-9.

Hara K, Yonezawa K, Weng QP, Kozlowski MT, Belham C, Avruch J. Amino acid sufficiency and mTOR regulate p70 S6 kinase and eIF-4E BP1 through a common effector mechanism. J Biol Chem. 1998;273(23):14484-14494.

Hsueh EC, Knebel SM, Lo WH, Leung YC, Cheng PNM, Hsueh CT. Deprivation of arginine by recombinant human arginase in prostate cancer cells. J Hematol Oncol. 2012;5:17.

Huang HL, Hsu HP, Shieh SC, Chang YS, Chen WC, Cho CY, et al. Attenuation of argininosuccinate lyase inhibits cancer growth via cyclin A2 and nitric oxide. Mol Cancer Ther. 2013;12(11):2505-16.

Huang HL, Chen WC, Hsu HP, Cho CY, Hung YH, Wang CY, et al. Argininosuccinate lyase is a potential therapeutic target in breast cancer. Oncol Rep. 2015;34(6):3131-9.

Husson A, Brasse-Lagnel C, Fairand A, Renouf S, Lavoinne A. Argininosuccinate synthetase from the urea cycle to the citrulline-NO cycle. Eur J Biochem. 2003;270:1887-99.

Izzo F, Marra P, Beneduce G, Castello G, Vallone P, De Rosa V, et al. Pegylated arginine deiminase treatment of patients with unresectable hepatocellular carcinoma: results from phase I/II studies. J Clin Oncol. 2004;22(10):1815-1822.

Jungbluth AA, Tassello J, Frosina D, Hanson N, Ritter G, $\mathrm{Wu}$ BW, et al. Expression pattern of ArgininosuccinateSynthetase (ASS) in normal and tumor tissue as a marker for susceptibility to Arginine-Deiminase (ADI) therapy. Mod Pathol. 2010;23(1):387A.
Kelly MP, Jungbluth AA, Wu BW, Bomalaski J, Old LJ, Ritter G. Arginine deiminase PEG20 inhibits growth of small cell lung cancers lacking expression of argininosuccinate synthetase. $\mathrm{Br}$ J Cancer. 2012;106(2):324-332.

Kim RH, Coates JM, Bowles TL, McNerney GP, Sutcliffe J, Jung JU, et al. Arginine deiminase as a novel therapy for prostate cancer induces autophagy and caspase-independent apoptosis. Cancer Res. 2009;69(2):700-708.

Kraemer PM. Interaction of mycoplasma (Pplo) and murine lymphoma cell cultures: prevention of cell lysis by arginine. Proc Soc Exp Biol Med. 1964;115:206-212.

Kuo MT, Savaraj N, Feun LG. Targeted cellular metabolism for cancer chemotherapy with recombinant arginine-degrading enzymes. Oncotarget. 2010;1(4):246-251.

Lam TL, Wong GK, Chong HC, Cheng PN, Choi SC, Chow TL, et al. Recombinant human arginase inhibits proliferation of human hepatocellular carcinoma by inducing cell cycle arrest. Cancer Lett. 2009;277(1):91-100.

Lam TL, Wong GK, Chow HY, Chong HC, Chow TL, Kwok $\mathrm{SY}$, et al. Recombinant human arginase inhibits the in vitro and in vivo proliferation of human melanoma by inducing cell cycle arrest and apoptosis. Pigment Cell Melanoma Res. 2010;24(2):366-376.

Leung YC, Wei XL. Arginine decarboxylase inhibits human colorectal cancer cells by inducing cell cycle arrest and apoptosis. Eur J Cancer. 2012;48(6):25-26.

Miraki-Moud F, Ghazaly E, Ariza-McNaughton L, Hodby KA, Clear A, Anjos-Afonso F, et al. Arginine deprivation using pegylated arginine deiminase has activity against primary acute myeloid leukemia cells in vivo. Blood. 2015;125(26):40604068.

Miyazaki K, Takaku H, Umeda M, Fujita T, Huang WD, Kimura $\mathrm{T}$, et al. Potent growth inhibition of human tumor cells in culture by arginine deiminase purified from a culture medium of a Mycoplasma-infected cell line. Cancer Res. 1990;50(15):45224527.

Morris SM. Arginine metabolism: boundaries of our knowledge. J Nutr. 2007;137(6):1602S-1609S.

Ni Y, Schwaneberg U, Sun ZH. Arginine deiminase, a potential anti-tumor drug. Cancer Lett. 2008;261(1):1-11. 
Nicholson LJ, Smith PR, Hiller L, Szlosarek PW, Kimberley $\mathrm{C}$, Sehouli J, et al. Epigenetic silencing of argininosuccinate synthetase confers resistance to platinum-induced cell death but collateral sensitivity to arginine auxotrophy in ovarian cancer. Int J Cancer. 2009;125(6):1454-1463.

Philip R, Campbell E, Wheatley DN. Arginine deprivation, growth inhibition and tumour cell death: 2. Enzymatic degradation of arginine in normal and malignant cell cultures. Br J Cancer. 2003;88(4):613-623.

Raijman L. Citrulline synthesis in rat tissues and liver content of carbamoyl phosphate and ornithine. Biochem J. 1974;138(2):225-232.

Roos D, Loos JA. Changes in the carbohydrate metabolism of mitogenically stimulated human peripheral lymphocytes. II. Relative importance of glycolysis and oxidative phosphorylation on phytohaemagglutinin stimulation. Exp Cell Res. 1973;77(1):127-35.

Rose WC, Haines WJ, Warner DT. The amino acid requirements of man. V. The rôle of lysine, arginine, and tryptophan. J Biol Chem. 1954;206(1):421-30.

Savaraj N, You M, Wu C, Wangpaichitr M, Kuo MT, Feun LG. Arginine deprivation, autophagy, apoptosis (aaa) for the treatment of melanoma. Curr Mol Med. 2010;10(4):405-412.

Sholler GLS, Gerner EW, Bergendahl G, MacArthur RB, VanderWerff A, Ashikaga T et al. A Phase I trial of DFMO targeting polyamine addiction in patients with relapsed/ refractory neuroblastoma. Plos One. 2015;10(5):e0127246.

Shuvayeva G, Bobak Y, Igumentseva N, Titone R, Morani F, Stasyk O, et al. Single amino acid arginine deprivation triggers prosurvival autophagic response in ovarian carcinoma SKOV3. BioMed Res Int. 2014;2014:ID505041.

Stone EM, Glazer ES, Chantranupong L, Cherukuri P, Breece $\mathrm{RM}$, Tierney DL, et al. Replacing $\mathrm{Mn}^{2+}$ with $\mathrm{Co}^{2+}$ in human arginase $\mathrm{i}$ enhances cytotoxicity towards 1-arginine auxotrophic cancer cell lines. ACS Chem Biol. 2010;5(3):333-342.

Tharakan JF, Yu YM, Zurakowski D, Roth RM, Young VR, Castillod L. Adaptation to a long term (4 weeks) arginine- and precursor (glutamate, proline and aspartate)-free diet. Clin Nutr. 2008;27(4):513-522.
Tomlinson BK, Thomson JA, Bomalaski JS, Diaz M, Akande T, Mahaffey N, et al. Phase I trial of arginine deprivation therapy with ADI-PEG 20 plus docetaxel in patients with advanced malignant solid tumors. Clin Cancer Res. 2015;21(11):24802486.

Tsai WB, Aiba I, Lee SY, Feun L, Savaraj N, Kuo MT. Resistance to arginine deiminase treatment in melanoma cells is associated with induced argininosuccinate synthetase expression involving c-Myc/HIF-1alpha/Sp4. Mol Cancer Ther. 2009;8(12):3223-3233.

Tytell AA, Neuman RE. Growth response of stable and primary cell cultures to 1-ornithine, 1-citrulline, and 1-arginine. Exp Cell Res. 1960;20(1):84-91.

Vynnytska-Myronovska B, Kurlishchuk Y, Bobak Y, Dittfeld C, Kunz-Schughart LA, Stasyk O. Three-dimensional environment renders cancer cells profoundly less susceptible to a single amino acid starvation. Amino Acids. 2013;45(5):1221-1230.

Vynnytska-Myronovska B, Bobak Y, Garbe Y, Dittfeld C, Stasyk O, Kunz-Schughart LA. Single amino acid arginine starvation efficiently sensitizes cancer cells to canavanine treatment and irradiation. Int J Cancer. 2012;130(9):2164-75.

Wangpaichitr M, Wu C, Bigford G, Theodoropoulos G, You M, Li YY, et al. Combination of arginine deprivation with TRAIL treatment as a targeted-therapy for mesothelioma. Anticancer Research. 2014;34(12):6991-6999.

Wheatley DN. Arginine deprivation and metabolomics: important aspects of intermediary metabolism in relation to the differential sensitivity of normal and tumour cells. Semin Cancer Biol. 2005;15(4):247-253.

Wu G, Morris SM Jr. Arginine metabolism: nitric oxide and beyond. Biochem J. 1998;336:1-17.

Yerushalmi HF, Besselsen DG, Ignatenko NA, Blohm-Mangone KA, Padilla-Torres JL, Stringer DE, et al. Role of polyamines in arginine-dependent colon carcinogenesis in Apc(Min) (/+) mice. Mol Carcinog. 2006;45(10):764-73.

Yoon CY, Shim YJ, Kim EH, Lee JH, Won NH, Kim JH, et al. Renal cell carcinoma does not express argininosuccinate synthetase and is highly sensitive to arginine deprivation via arginine deiminase. Int J Cancer. 2007;120(4):897-905. 
Yoon JK, Frankel AE, Feun LG, Ekmekcioglu S, Kim KB. Arginine deprivation therapy for malignant melanoma. Clin Pharmacol. 2013;5:11-19.

Yu YM, Ryan CM, Castillo L, Lu XM, Beaumier L, Tompkins $\mathrm{RG}$, et al. Arginine and ornithine kinetics in severely burned patients: increased rate of arginine disposal. Am J Physiol Endocrinol Metab. 2001;280(3):E509-17.
Zeng X, Li Y, Fan J, Zhao H, Xian Z, Sun Y, et al. Recombinant human arginase induced caspase-dependent apoptosis and autophagy in non-Hodgkin's lymphoma cells. Cell Death Dis. 2013;4:e840.

Received for publication on $22^{\text {th }}$ November 2016 Accepted for publication on $15^{\text {th }}$ May 2017 